\title{
Classical statistical thermodynamics of a gas of charged particles in magnetic field
}

\author{
I.M.Dubrovskii \\ Institute for Metal Physics \\ 36 Vernadsky Str., Kyiv 03680, Ukraine
}

Received July 4, 2005

We will demonstrate that the paradox of classical statistical thermodynamics for a gas of charged particles in a magnetic field (GMF) has not yet been explained. The paradox lies in the statement that the average magnetic moment of a gas is zero, whereas the time-average magnetic moment of each particle is always negative. We consider a gas of charged particles moving in a plane perpendicular to a uniform magnetic field. The density of distribution of the ensemble describing statistical properties of the GMF is derived starting from the basics, with due regard for the specific character of dynamics of the charged particles in the magnetic field. It is emphasized that neither the imposition of a potential barrier restricting the existence region of the GMF, nor the introduction of a background neutralizing charge occupying a finite area, is a necessary condition for the stationary equilibrium state of the GMF to exist. We show that the reason for this fact is that the density of distribution of the ensemble is dependent, besides the Hamiltonian, on another positive definite integral of motion that is a linear combination of the Hamiltonian and the angular momentum of the GMF. Basic thermodynamic relations are deduced in terms of the new density of distribution, and it is demonstrated that the GMF has a magnetic moment whose magnitude and sign are determined by the external potential field. Particularly, the GMF is diamagnetic in the absence of the neutralizing background charge. Thus, the statement of the Bohr-van Leeuwen theorem, deduced using the ordinary Gibbs density of distribution depending on the Hamiltonian only, is wrong. It is noted that a great deal of works on the theory of electronic phenomena in magnetic field are based either on the same wrong density of distribution or on the formula for average occupation numbers depending on the energy of states, which follows from this density of distribution within quantum theory. These theories should be revised in view of the new form of the density of distribution.

Key words: gas, charged particles, magnetic field, density of distribution, phase space, integral of motion, thermodynamics, magnetic moment

PACS: $05.20 . \mathrm{Gg}, 75.20 .-\mathrm{g}$

\section{Introduction}

"Finally, it is shown that the presence of free electrons, contrary to the generally adopted opinion, will not give rise to any magnetic properties of the metals". This sentence ends a short report [1] on the presentation "Electron theory of metals" by N. Bohr, given at the meeting of the Philosophical Society at Cambridge. This presentation was a survey of his dissertation [2]. The other results presented in the dissertation confirmed as well as improved the Lorentz theory; however, the above result appeared to be paradoxical. It was well-known that a charged particle in a uniform magnetic field moves in such a way that the time average of the magnetic moment, generated by this motion, is directed opposite to the magnetic field. A gas of such particles seemed to have a negative moment, explaining the diamagnetism of some metals.

N. Bohr described statistical properties of a gas of charged particles in a magnetic field (GMF) using the Gibbs density of distribution given by

$$
f_{N}(p, q)=Z^{-1} \exp [-\mathrm{H}(p, q) / T],
$$

whose domain of definition in the coordinate subspace of the phase space is restricted by the condition that all the particles are located within a definite volume $V$. Here $N$ is the number of 
particles, $(p, q)$ denotes the set of all momenta and coordinates of particles of the gas,

$$
Z=\int_{\Phi_{p}} \int_{\Phi_{q}} \exp [-\mathrm{H}(p, q) / T] \mathrm{d} \Phi_{p} \mathrm{~d} \Phi_{q}
$$

is the partition function, $\Phi_{p}$ and $\Phi_{q}$ are momentum and coordinate subspaces of the phase space, respectively, $T$ is temperature, and $\mathrm{H}(p, q)$ is the Hamiltonian of the GMF given by

$$
\mathrm{H}(p, q)=\sum_{\nu} w_{\nu}+U, \quad w_{\nu}=\frac{1}{2 m}\left[\left(p_{\nu x}-\frac{\mathrm{e}}{2 c} H y_{\nu}\right)^{2}+\left(p_{\nu y}+\frac{\mathrm{e}}{2 c} H x_{\nu}\right)^{2}\right] .
$$

For simplicity, we will restrict ourselves to the case of a two-dimensional gas on a plane, perpendicular to the uniform magnetic field $\mathbf{H}=(0,0, H)$. Throughout this paper, where it is needed, we imply the plane to be of finite "thickness" $\delta z$, and, for example, the equations of electrodynamics are written for three-dimensional space. In (3) we use Cartesian coordinates and corresponding momenta. The potential energy $U$ of the system describes the interaction of particles among themselves and with an external potential field. It depends on the coordinates of particles only. The charge of a particle - $\mathrm{e}$ is assumed to be negative, $m$ is the particle mass, and $c$ is the speed of light in a vacuum. Summation over $\nu$ denotes the summation over all particles composing the gas. We choose the origin of coordinates to be in the mass center of the gas and assume it to be fixed with respect to the conductors containing the current generating the magnetic field.

If the partition function as a function of volume, temperature and magnetic field is known, then all the thermodynamic quantities, characterizing the GMF, are defined. Then Bohr's paradoxical statement is simple to prove. In the Cartesian coordinates, integration over the momentum space is carried out with infinite limits. Changing the integration variables by

$$
p_{\nu x}-(\mathrm{e} H / 2 c) y_{\nu}=\pi_{\nu x}, \quad p_{\nu y}+(\mathrm{e} H / 2 c) x_{\nu}=\pi_{\nu y}
$$

we find that the partition function is independent of the magnetic field. Bohr's "Dissertation" has not been published. A variant of the above proof was presented by van Leeuwen [3], and the statement about the absence of magnetic properties in the GMF is known as the Bohr-van Leeuwen theorem. This issue is extensively discussed in the monograph by van Vleck [4]. In the literature there were many attempts to explain the mechanisms of the absence of magnetic properties in the GMF. The most popular explanation is that a current, created by a drift of near-boundary orbits, generates a paramagnetic moment compensating for the total diamagnetic moment of interior orbits. No comprehensive computation of such a compensation is available in literature, even as an example. An attempt to perform such a computation suggests that a value for the boundary current does not directly follow from equations (1) and (3), and that its calculation requires a number of voluntary assumptions. R. Peierls [5] was astonished at the way the boundary current, depending apparently on a boundary shape, a character of particle reflection, etc., was capable of completely compensating for the diamagnetism of the interior electrons.

Although no convincing treatment of the paradox has been proposed, none has cast any doubt on the validity of the content of the Bohr-van Leeuwen theorem. This statement was referred to in each academic course or review monograph where the magnetism of the electron gas in conductors was considered (see, e.g. [4-11]). Nobody cast doubt on the validity of the application of the density of distribution (1) and its quantum analogue to the description of statistical properties of the GMF. The diamagnetism of metals remained unexplained within the framework of classical statistical thermodynamics.

In 1930 L. D. Landau proposed an explanation of the diamagnetism of metals as a quantum phenomenon [12]. To calculate the thermodynamic potential, he used the quantum analogue of the density of distribution (1) (statistical operator) and a formula for an average occupation number of a state depending on its energy only (Fermi distribution), which follows immediately from (1). As will be shown in future work, the Landau theory contains some discrepancies and unjustified assumptions, caused by poor level of quantum mechanics and a lack of some special mathematical knowledge at that time. As a result, a term in the thermodynamic potential was ignored. This 
term would give a paramagnetic contribution to the magnetic moment that by far exceeds the diamagnetic contribution taken into account in [12]. A consistent application of the quantum analogue of the density of distribution (1) to the calculation of the magnetic moment of the GMF yields a result incompatible with experimentally observed facts.

The aim of the present study is to demonstrate that the use of the density of distribution (1) to describe the statistical properties of the GMF is not logically relevant, because it does not properly take into account the specific character of the dynamics of a charged particle in a magnetic field, which are different from the dynamics of particles in the inertial motion or moving under the action of a potential field. It will be shown that, besides the Hamiltonian, there is another global, and positive definite integral of motion of the GMF. Together with the Hamiltonian, this integral of motion restricts completely an area of the phase space where the whole trajectory of the GMF lies. Therefore, the density of distribution of an ensemble, describing statistical properties of the GMF, should depend not only on the Hamiltonian but also on this integral of motion.

In section 2 we give a description of the classical dynamics of the GMF taking into account its essential features which were previously discarded in the statistical theory.

In section 3 we develop and justify a new approach to the statistical description of the classical GMF, derive the density of distribution for the canonical ensemble, and obtain basic thermodynamic relations. This density of distribution will be used to demonstrate that the GMF can possess either positive or negative magnetic moment, depending on the magnitudes of external parameters. The quantum statistical mechanics of the GMF will be the subject of the follow-up work.

\section{Dynamic description of a gas of particles in a magnetic field}

Here we recall the basic properties of motion of a charged particle in a magnetic field and introduce some new motion characteristics and formulae required for a further consideration. A particle traces out a stationary circular orbit of radius $\rho=\sqrt{2 \varepsilon / m \omega^{2}}$, where $\varepsilon$ is the particle energy, $\omega=\mathrm{e} H / m c$ is the circular frequency, and $T_{0}=2 \pi / \omega$ is the period of revolution. The Cartesian coordinates of the orbit center are the integrals of motion:

$$
X=-c p_{y} / \mathrm{e} H+x / 2, \quad Y=c p_{x} / \mathrm{e} H+y / 2
$$

If a force $\mathbf{F}(x, y)$ acts on a particle, its motion can be described as rotation about the center moving with the velocity

$$
\mathbf{V}=(\dot{X}, \dot{Y})=-\left(c / \mathrm{e} H^{2}\right)[\mathbf{F} \times \mathbf{H}]
$$

Such a motion of the orbit center is said to be a drift. If the force $\mathbf{F}(x, y)$ results from the action of a potential field $u(r)$, symmetrical relative to the point of origin, with $r=\sqrt{x^{2}+y^{2}}$, the angular momentum of a particle about $z$-axis is the other integral of motion. It is defined as

$$
l_{z}=x p_{y}-y p_{x}=\frac{w}{\omega}-\frac{m \omega R^{2}}{2}, \quad R^{2}=X^{2}+Y^{2} .
$$

For the purpose of the following consideration, it is needed to consider a linear combination of the angular momentum and the Hamiltonian $\mathrm{h}=w+u$ as an integral of motion. Let us denote this integral of motion by $\mathrm{d}$ and call it the $d$-integral,

$$
\mathrm{d}=\mathrm{h}-\omega l_{z}=u(r)+\frac{m \omega^{2} R^{2}}{2} .
$$

If the energy reference is chosen so that the potential energy $u(r)$ is positive throughout, then both the Hamiltonian and the $d$-integral are positively defined values. As a result, as it will be shown later, the use of the conservation law for the $d$-integral has an advantage over the angular momentum. As it follows from equation (7), the sign of the angular momentum is determined by the relation between the orbit radius, proportional to the square root of the kinetic energy value, and the distance of its center to the origin. 
The similarity between the Hamiltonian and the $d$-integral is more evident, if one goes on to new canonical variables. It was noticed in [12] that the Poisson bracket of the Cartesian components of the velocity of a particle in a magnetic field is equal to a constant, so that the values proportional to these components may be chosen as a new pair of canonical variables, which should be denoted as $\left(P_{h}, Q_{h}\right)$. The second pair of the canonical variables $\left(P_{d}, Q_{d}\right)$ is represented by the values proportional to the Cartesian coordinates of the orbit center in the absence of the potential field (5). The generating function of the canonical transformation appears as

$$
F\left(Q_{h}, Q_{d} ; x, y\right)=\frac{\mathrm{e} H}{c}\left(Q_{h} Q_{d}-Q_{d} y-Q_{h} x+\frac{1}{2} x y\right), \quad p_{i}=\frac{\partial F}{\partial i}, \quad P_{j}=-\frac{\partial F}{\partial Q_{j}} .
$$

Here $i$ takes on the values $x, y$, and $j$ takes on $d, h$. The new variables are related to the old ones by

$$
\begin{array}{llrl}
P_{h} & =p_{y}+\mathrm{e} H x / 2 c=m \nu_{y}, & & Q_{h}=-c p_{x} / \mathrm{e} H+y / 2=-\nu_{x} / \omega \\
P_{d}=p_{x}+\mathrm{e} H y / 2 c=\mathrm{e} H Y / c, & Q_{d}=-c p_{y} / \mathrm{e} H+x / 2=X .
\end{array}
$$

In terms of the new variables, the integrals of motion take on the form

$$
\begin{aligned}
\mathrm{h} & =\frac{P_{h}^{2}}{2 m}+\frac{m \omega^{2} Q_{h}^{2}}{2}+u(r)=w_{h}+u(r), \\
\mathrm{d} & =\frac{P_{d}^{2}}{2 m}+\frac{m \omega^{2} Q_{d}^{2}}{2}+u(r)=w_{d}+u(r), \\
r^{2} & =x^{2}+y^{2}=Q_{d}^{2}+Q_{h}^{2}+\frac{1}{m^{2} \omega^{2}}\left(P_{d}^{2}+P_{h}^{2}\right)+\frac{2}{m \omega}\left(P_{d} Q_{h}+P_{h} Q_{d}\right) .
\end{aligned}
$$

Exchange of indices $d \rightleftarrows h$ makes the integrals of motion $\mathrm{h}$ and $\mathrm{d}$ transform one into another, while the potential energy $u(r)$ is invariant under such exchange.

The magnetic moment of a particle,

$$
m_{z}=-\frac{\mathrm{e}}{2 c}\left(x \frac{\partial \mathrm{h}}{\partial p_{y}}-y \frac{\partial \mathrm{h}}{\partial p_{x}}\right)=-\frac{\partial \mathrm{h}}{\partial H}
$$

in contrast to the angular momentum, is not an integral of motion. In the absence of the potential field, the value of the magnetic moment averaged over the period $T_{0}$ for one particle is

$$
\left\langle m_{z}\right\rangle_{T_{0}}=-\varepsilon / H
$$

where the energy of the particle $\varepsilon$ is equal, in this case, to its kinetic energy. This means that, if a particle moving on its orbit is replaced by a circular current, its energy is represented as the energy of the current in the magnetic field.

In the potential field described above, the force exerted on a particle has a radial component only. The drift velocity (6) has both azimuthal and radial components, provided the azimuths of the instantaneous position of the particle and its orbit center do not coincide. Therefore, the distance $R$ of the orbit center to the origin of coordinates is not conserved in the potential field. If the gradient direction of the potential field is constant throughout the area of motion, then the azimuthal component of the drift velocity does not reverse its sign, while the radial one does, passing through zero at the moments when the particle and its orbit center are at the same radius. The trajectory, on the whole, can appear as a complicated curve, but it is confined between concentric circles, and the motion is conditionally periodic.

Let us examine the changes in the motion of a particle when the magnetic field, remaining homogeneous, varies uniformly by the value of $\delta H$ in time period $\delta t$. We also assume the current, generating the magnetic field, to be axially symmetric. Then the variation of the magnetic field generates an electric field that has only an azimuthal component and is expressed as

$$
E_{\varphi}=-\frac{r}{2 c} \frac{\delta H}{\delta t}
$$


At $\delta H \ll H$, the work done by the field on the particle, i.e. the change of the particle energy $\varepsilon$ due to the magnetic field variation, in the linear approximation with respect to $\delta H / H$, is given by

$$
\delta \varepsilon=-\mathrm{e} \int_{0}^{\delta t}(\mathbf{E} \cdot \mathbf{v}) \mathrm{d} t=\frac{\mathrm{e}}{2 c}\left|\frac{1}{\delta t} \int_{0}^{\delta t}[\mathbf{r} \times \mathbf{v}] \mathrm{d} t\right| \delta H=-\left\langle m_{z}\right\rangle_{\delta t} \delta H .
$$

Here, the second expression was obtained using equation (14). Hence, it follows that the work done is proportional, firstly, to the algebraic sum of the area swept out by the radius-vector of the particle in the time $\delta t$, i.e. to the average magnetic moment, and, secondly, to the variation of the magnetic field $\delta H$. The same result can also be obtained by integration of the total time derivative of the Hamiltonian, with the changing magnetic field and in view of equation (12). For a particle in the absence of a potential field one can write

$$
\delta \varepsilon=\frac{\varepsilon}{H} \delta H
$$

Similarly, we calculate an increment $\delta \beta$ of the value of the $d$-integral, which we will denote by $\beta$, due to the drift under the effect of the field (14). We obtain

$$
\begin{aligned}
& \delta \beta=m \omega^{2} R(\mathrm{~d} R)_{E}=m \omega^{2} R \int_{0}^{\delta t} V_{E} \frac{(\mathbf{R} \cdot \mathbf{r})}{R r} \mathrm{~d} t=\frac{m \omega^{2}}{2 H} \frac{\delta H}{\delta t} \int_{0}^{\delta t}(\mathbf{R} \cdot \mathbf{r}) \mathrm{d} t \\
& \text { or } \\
& \delta \beta=\int_{0}^{\delta t} \frac{\mathrm{dd}}{\mathrm{d} t} \mathrm{~d} t=\frac{\delta H}{\delta t} \int_{0}^{\delta t} \frac{\partial \mathrm{d}}{\partial H} \mathrm{~d} t=\frac{m \omega^{2}}{2 H} \frac{\delta H}{\delta t} \int_{0}^{\delta t}(\mathbf{R} \cdot \mathbf{r}) \mathrm{d} t
\end{aligned}
$$

because the $d$-integral depends only on time through the time dependence of the magnetic field. Here subscript $E$ means that we consider only the values determined by the vortex electric field (14), i.e. $V_{E}$ is expressed by equation (6), where the force has only an azimuthal component $F_{\varphi}=-\mathrm{e} E_{\varphi}$. If there is no potential field, we get

$$
\delta \beta=\frac{\beta}{H} \delta H,
$$

similarly to (16). A consequence of the $d \rightleftarrows h$ symmetry is the possibility to introduce, along with the magnetic moment of the particle,

$$
m_{z(h)}=-\frac{\partial \mathrm{h}}{\partial H}=-\frac{w_{h}}{H}-\frac{\omega}{2 H}\left(P_{h} Q_{d}+P_{d} Q_{h}\right)
$$

a similar quantity $m_{z(d)}=-\partial \mathrm{d} / \partial H$, describing, according to (17), the variation of the $d$-integral due to a change of the magnetic field. In the new variables, the functional form of $m_{z(d)}$ is the same as that of (19), up to the exchange of indices $d$ and $h$.

Now, let us consider a gas of charged particles and start with the assumption that the gas is ideal, i.e. the interaction between particles is represented only by their repulsion at collisions. The repulsion is not incorporated into the Hamiltonian because it is substantial only within a small fraction of the phase space volume. However, it is believed to be the cause for the gas dynamics to become chaotic. Such a model is not realistic, but (with an external field added in the form of infinitely high bounding walls) it is considered in [12] and in a great deal of other works on the quantum statistical theory of the GMF. The physical meaning of the results obtained within this model will be discussed in section 3 .

A collision of two round particles is accompanied by the conservation of a sum of their energies and a sum of values of their $d$-integrals. As seen from equation (6), the centers of the orbits of the collided particles are shifted by equal distances in opposite directions. Based on the laws of conservation, one can demonstrate that positions of the orbit centers of the particles before and after the collision form a rectangle. As the particle radius approaches zero, the direction of shifts of the orbit centers tends to a bisectrix of the angle between the tangents to the orbits at the intersection point. Which of the two bisectrices, each perpendicular to the other, will define this 
direction, depends upon a small phase difference of the particles motion on the orbits. In the motion of the whole system possessing $2 N$ degrees of freedom, two global single-valued positive definite integrals,

$$
\mathrm{H}=\sum_{\nu} w_{\nu}=E, \quad \mathrm{D}=\sum_{\nu} d_{\nu}=\frac{m \omega^{2}}{2} \sum_{\nu} R_{\nu}^{2}=B,
$$

are conserved, where $E$ and $B$ are constants. Since the value of the total energy is proportional to a sum of the squares of the radii of the orbits described by the particles, while the value of the total $d$-integral is proportional to the sum of the squares of the distances from their centers to the mass center of the GMF, it is obvious that the conservation laws forbid an unlimited spreading of the GMF over the plane. As it is known, the collision processes should give rise to the Maxwell distribution of values of the kinetic energy of particles. In view of the parallels between the kinetic energy and the total $d$-integral, a similar distribution of values $R_{\nu}^{2}$ should be also realized. A more detailed consideration of this situation will be given in section 3 .

In the case of a gas of particles that repulse each other, not only at a direct contact, but also according to Coulomb's law, we may again introduce an integral of motion D by

$$
\mathrm{D}=\mathrm{H}-\omega \mathrm{L}_{z}=\frac{m \omega^{2}}{2} \sum_{\nu} R_{\nu}^{2}+U, \quad \mathrm{~L}_{z}=\sum_{\nu} l_{z, \nu}=\sum_{\nu}\left(\frac{w_{\nu}}{\omega}-\frac{m \omega R_{\nu}^{2}}{2}\right),
$$

because the central interaction does not break the axial symmetry of the system, so the total angular momentum is an integral of motion. However, in this case the individual quantities $R_{\nu}^{2}\left(p_{\nu}, q_{\nu}\right)$, as well as $w_{\nu}\left(p_{\nu}, q_{\nu}\right)$, are not integrals of motion. In the case of a long-range interaction, neither the $d$-integral, nor the Hamiltonian can be represented as sums of the corresponding integrals of motion for individual particles. From equations (10) we see that the exchange of indices of the new canonical variables $d \rightleftarrows h$ corresponds to the exchange of the Cartesian coordinates $x \rightleftarrows y$. Therefore, the potential energy of the interacting charged particles is invariant with respect to the $d \rightleftarrows h$ exchange, while the Hamiltonian and the $d$-integral change one into another with this exchange. Since the potential energy $U\left(\left\{\mathbf{r}_{\nu}\right\}\right)$, depending on the complete collection of the coordinates of the particles $\left\{\mathbf{r}_{\nu}\right\}$, is everywhere positive, equations (3) and (21) imply that the conservation laws forbid an infinite spreading of the gas over the plane even in the case of the Coulomb repulsion between particles.

Since the GMF remains within a restricted domain of the plane, defined by initial conditions, during an infinitely long time, its motion should be either periodic or chaotic. It is commonly agreed that the motion of a gas, where collisions occur, is chaotic. The collisions cannot vanish with time because this would imply a change-over to the periodic motion; this is impossible because a motion being periodic in the future is necessarily periodic in the past.

\section{Statistical thermodynamics of a gas of charged particles in a magnetic field}

Let us proceed from an experimentally proven fact that a stationary equilibrium state can be realized in an isolated GMF or in a GMF being in contact with a thermostat. To study the properties of such a state, we use the methods of statistical mechanics. The results of the preceding section suggest that a phase trajectory of an isolated GMF in the $4 N$-dimensional phase space of variables (10), that runs over the hypersurface

$$
\mathrm{H}(P, Q)=E, \quad \mathrm{D}(P, Q)=B
$$

remains within a bounded domain of the phase space. This statement is also valid for the phase space in the Cartesian coordinates and the corresponding momenta of the particles. For many systems generally considered in the literature, e.g. for a gas of rigid disks which move constantly in the time between collisions, this rule does not hold, even if the total azimuthal moment is conserved, and such a gas should expand infinitely with time. 
The GMF does not possess the properties of additivity and uniformity (i. e. the assumption that the system is composed of macroscopic parts, which are approximately noninteracting, each of them being similar in its properties to the whole system), even if the long-range repulsion is discarded. As it was shown earlier, the area in which $N$ particles were located at the initial moment cannot change substantially with time. Therefore, both the motion and collisions of particles should result in the formation and conservation of inhomogeneous, axially symmetric density of particles and an energy distribution of particles. To describe the properties of an electron gas in a magnetic field, one needs a statistical theory of this stationary equilibrium state of the GMF. Due to a lack of the properties of additivity and uniformity, the notion of thermodynamic limit is inapplicable. Such external parameters as "system volume (area)" and "pressure", related to one another, are not considered as mandatory parameters. Bounding the area by an infinite potential barrier or the inclusion of a neutralizing charge of homogeneous density, resulting also in the appearance of the finite potential barrier, brings additional complications to the model, which are not necessary for the stationary state to be established. Instead, another external parameter, the magnetic field strength $H$, becomes mandatory. Therefore, we should start to construct the statistical theory of the GMF with the basics.

In [6], the choice of the integrals of motion, which the density of distribution must be dependent, is based on the additivity property. Next, three of four (for two-dimensional case) integrals of motion, the components of the momentum and the angular momentum, are discarded, the justification for this operation being a non-rigorous proof. It was supposed that the logarithm of the density of distribution of the system could be represented as a sum of logarithms of the distribution densities of its component subsystems. However, the density of distribution of the microcanonical ensemble proposed later is apparently inconsistent with this assumption. The matter is that, in the case of a microcanonical ensemble, the integrals of motion do not define a probability of a state as a function of coordinates in the phase space, but they determine an area where the density of distribution is equal to a non-zero constant. The area of the phase space, where the density of distribution is constant, is a direct product of such areas for the subsystems, because the phase space of the system is a direct product of the phase spaces of the subsystems.

In [13] this area was taken a priori to be defined by both the restriction of the system volume and global, unique and additive integrals of motion, of which the energy only is retained. To compute the volume of this area, a method proposed by Yu.A. Krutkov [14] is used, with the assumption that the Hamiltonian of the system is represented as a sum of a large number of identical Hamiltonians of particles or subsystems with negligible interactions. Yu.A. Krutkov appealed to P. Debye [15], whose idea was used as the basis for the method. In the resulting formula, the volume of the phase space area, where all the systems composing the microcanonical ensemble are distributed with a constant density, is written as a function of energy, number of particles, and volume occupied by the system. This expression is a fundamental formula in statistical mechanics. It is usually applied to any system in a stationary equilibrium state, including the systems which do not satisfy the assumptions made in its proof. R. Kubo [16] just postulated this expression without proof, and the systems for which it holds are referred to as normal in the sense of statistical thermodynamics. From the same formula one can deduce the Gibbs distribution (1), assuming that a system considered is a subsystem of a medium of similar structure, being separated from it by a partition, and allowing for collisions of particles, but not for their exchanges. If one considers a system of interacting particles in the mean field approximation, it should be assumed that the partition also screens the field formed by a thermostat.

The fact that the microcanonical distribution is determined only by the energy integral was explained by A.Ya. Hinchin in his monograph [17]. The integrals of momentum and angular momentum do not restrict the volume in the phase space or in its momentum or coordinate subspaces. The existence of such a restricted volume, determined by the laws of conservation, is a necessary condition for the stationary equilibrium state. The energy is a positive definite quantity, increasing monotonically with the module of any momentum in the system. Hence, the constant energy surface restricts a constant-volume domain of the momentum subspace, with the phase trajectory remaining within a phase space layer bounded by the surfaces defined by $\mathrm{H}(P, Q)=E$ and 
$\mathrm{H}(P, Q)=E+\Delta E$ during an infinitely long period. In the coordinate subspace, the volume is restricted by the condition that particles do not leave the system. The consideration of the laws of conservation of total momentum and angular momentum can only incidentally alter the volume of this domain.

If we change to new canonical variables (10) in equations (3), (21) similarly to (11), we can see that all the arguments suitable to the Hamiltonian $\mathrm{H}$ in the case of the GMF, are also suitable to the $d$-integral $\mathrm{D}$. Therefore, in this case the phase trajectory remains within the phase space layer of a finite volume bounded by the surfaces defined by equations $(\mathrm{H}=E, \mathrm{D}=B)$ and $(\mathrm{H}=E+\Delta E, \mathrm{D}=B+\Delta B)$ during an infinitely long period. Thus, in the case of the GMF, it is necessary to change the formulation of the principal hypothesis of statistical mechanics. Let us suppose that macroscopic properties of an isolated GMF system can be represented by a statistical ensemble whose density of distribution is constant within this layer and is zero beyond it. As usual, we ignore the fact that a part of the layer volume is occupied with the paths of the periodic motion of the system, assuming this part to be negligible.

The methods, currently recognized for the calculation of the volume of such a layer, use the possibility for the Hamiltonian (in our case, for both the Hamiltonian and the $d$-integral) to be represented as a sum of a large number of the Hamiltonians (and the $d$-integrals) for non-interacting systems or particles. The absence of the interaction implies that the latter is so small, or different from zero within such a small area of the phase space, that it can be neglected in the Hamiltonian. As an example of such an interaction, we refer to collisions of particles in a gas. Nevertheless, the interaction is sufficient to make the motion chaotic. The statistical description cannot be applied to a gas of absolutely non-interacting particles.

If the Coulomb interaction in the GMF is taken exactly into account, the Hamiltonian and the $d$-integral cannot be represented by such sums. Let us assume the Coulomb repulsion of particles in the equilibrium to be approximately described by the combination of an average axially symmetric electric field, vanishing at large distances from the origin, and rigid repulsion of round particles at collisions. This is a central interaction, so that the total angular momentum with respect to the origin is conserved. We may also assume the presence of a uniform background field of neutralizing charge, filling the area inside a circle of radius $R_{N}$. Interaction of particles with the background can also be described in terms of a mean field, with the energy reference chosen so that the potential energy of a particle in the mean field is everywhere non-negative. It is customary to implicitly suppose that the particles uniformly fill the entire circle of radius $R_{N}$, and, hence, to assume the potential of the total mean field to be identically zero inside the circle bounded by a high potential barrier. In fact, as it will be shown below, the assumption of a uniform distribution of particles in the stationary equilibrium state does not hold. The potential of a self-consistent mean field should have a maximum at the center of the circle, should be minimal in the vicinity of the boundary, and should tend to a constant value beyond the circle.

In this situation, to calculate a phase volume where the density of distribution is nonzero, let us generalize the method proposed by Yu. Krutkov [14]. A similar, in its essence, approach was also used in [17]. A volume of the phase space confined within the surface (22) is represented as

$$
V(E, B)=\int_{\Phi} \mathrm{d} \Phi \Theta(E-\mathrm{H}) \Theta(B-\mathrm{D})
$$

where integration is done over the whole phase space, $d \Phi$ is its element, and $\Theta(a-x)$ is the step-function

$$
\Theta(a-x)= \begin{cases}1, & x<a \\ 0, & x>a\end{cases}
$$

To define a layer near the surface (22), it is necessary to assume $\Delta E \ll E$ and $\Delta B \ll B$. In all other respects, specific values of $\Delta E$ and $\Delta B$ are unessential, so we take $\Delta B=\Delta E=\Delta$. Let $\Gamma(E, B)$ be the layer volume given by

$$
\Gamma(E, B)=\left(\frac{\partial V}{\partial E}+\frac{\partial V}{\partial B}\right) \Delta .
$$


Now, let us calculate the Laplace transformation of $\Gamma(E, B)$ with respect to both variables. The first term is

$$
\begin{aligned}
f_{h} & =\int_{0}^{\infty} \mathrm{d} E \int_{0}^{\infty} \mathrm{d} B \frac{\partial V}{\partial E} \exp \left(-\lambda_{h} E-\lambda_{d} B\right) \\
& =\int_{0}^{\infty} \mathrm{d} E \int_{0}^{\infty} \mathrm{d} B \exp \left(-\lambda_{h} E-\lambda_{d} B\right) \int_{\Phi} \mathrm{d} \Phi \delta(E-\mathrm{H}) \Theta(B-\mathrm{D}) .
\end{aligned}
$$

Substitute

$$
\Theta(B-\mathrm{D})=\int_{0}^{B} \delta(z-\mathrm{D}) \mathrm{d} z
$$

into (26) and integrate over $B$ by parts. We get

$$
\begin{aligned}
f_{h} & =\frac{1}{\lambda_{d}} \int_{0}^{\infty} \mathrm{d} E \int_{0}^{\infty} \mathrm{d} B \exp \left(-\lambda_{h} E-\lambda_{d} B\right) \int_{\Phi} \mathrm{d} \Phi \delta(E-\mathrm{H}) \delta(B-\mathrm{D}) \\
& =\frac{1}{\lambda_{d}} \int_{\Phi} \mathrm{d} \Phi \exp \left(-\lambda_{h} \mathrm{H}-\lambda_{d} \mathrm{D}\right)=\frac{1}{\lambda_{d}}\left[f_{0}\left(\lambda_{h}, \lambda_{d}\right)\right]^{N},
\end{aligned}
$$

where a function was introduced,

$$
f_{0}\left(\lambda_{h}, \lambda_{d}\right)=\int_{\Phi_{\nu}} \mathrm{d} \Phi_{\nu} \exp \left(-\lambda_{h} \mathrm{~h}_{\nu}-\lambda_{d} \mathrm{~d}_{\nu}\right)
$$

identical for all particles. In this calculation, we used the representations of the Hamiltonian and the $d$-integral as sums with respect to all the particles, and the relation $\mathrm{d} \Phi=\prod_{\nu} \mathrm{d} \Phi_{\nu}$. The image of the second term in (25) is calculated similarly. The image of the phase volume $\Gamma(E, B)$ appears as

$$
f\left(\lambda_{h}, \lambda_{d}\right)=\left(\frac{1}{\lambda_{h}}+\frac{1}{\lambda_{d}}\right)\left[f_{0}\left(\lambda_{h}, \lambda_{d}\right)\right]^{N} \Delta .
$$

Thus, at large $N$, one is allowed to employ the saddle-point method in making the inverse Laplace transformation to calculate the phase volume. We have

$$
\Gamma(E, B)=\Delta\left(\frac{1}{2 \pi i}\right)^{2} \int_{a_{h}-i \infty}^{a_{h}+i \infty} \mathrm{d} \lambda_{h} \int_{a_{d}-i \infty}^{a_{d}+i \infty} \mathrm{d} \lambda_{d}\left(\frac{1}{\lambda_{h}}+\frac{1}{\lambda_{d}}\right) \exp \left[N \chi\left(\lambda_{h}, \lambda_{d} ; E, B\right)\right]
$$

where

$$
\chi\left(\lambda_{h}, \lambda_{d} ; E, B\right)=\lambda_{h} \frac{E}{N}+\lambda_{d} \frac{B}{N}+\ln f_{0}\left(\lambda_{h}, \lambda_{d}\right) .
$$

The potential energy $u(r)$ of a particle tends to zero or to a finite positive number at $r \rightarrow \infty$. Then it follows from equations (11) that the value of the integral (28) tends to infinity as either $\lambda_{h}$ or $\lambda_{d}$ approaches zero. Therefore, $\chi$, as a function of these parameters, tends to infinity as either of them tends to zero or to infinity, thus, it has a minimum at a point with positive coordinates $\left(\Lambda_{h}, \Lambda_{d}\right)$ satisfying the following relations:

$$
\left\{\begin{array}{l}
{\left[\frac{\partial \chi}{\partial \lambda_{h}}\right]_{\substack{\lambda_{h}=\Lambda_{h} \\
\lambda_{d}=\Lambda_{d}}}=\frac{E}{N}+\frac{1}{f_{0}\left(\Lambda_{h}, \Lambda_{d}\right)}\left[\frac{\partial f_{0}}{\partial \lambda_{h}}\right]_{\substack{\lambda_{h}=\Lambda_{h} \\
\lambda_{d}=\Lambda_{d}}}=0} \\
{\left[\frac{\partial \chi}{\partial \lambda_{d}}\right]_{\substack{\lambda_{h}=\Lambda_{h} \\
\lambda_{d}=\Lambda_{d}}}=\frac{B}{N}+\frac{1}{f_{0}\left(\Lambda_{h}, \Lambda_{d}\right)}\left[\frac{\partial f_{0}}{\partial \lambda_{d}}\right]_{\substack{\lambda_{h}=\Lambda_{h} \\
\lambda_{d}=\Lambda_{d}}}=0}
\end{array}\right.
$$

The parameters $\Lambda_{h}$ and $\Lambda_{d}$ are functions of $E / N$ and $B / N$, i.e. they are internal parameters. The GMF can be considered as an axially symmetric charged body. Then, in the magnetic field its mass center should describe a circular orbit, and the angular momentum of the GMF as a whole is nonzero. The assumption that the mass center is fixed with respect to conductors containing the current generating the magnetic field, is equivalent to the assumption that the angular momentum 
of the GMF is zero. Then $E=B$ and $\Lambda_{h}=\Lambda_{d}$. Choosing $\Lambda_{h}$ and $\Lambda_{d}$ as saddle points to evaluate the integrals in equation (30), we obtain (the order of integration does not matter):

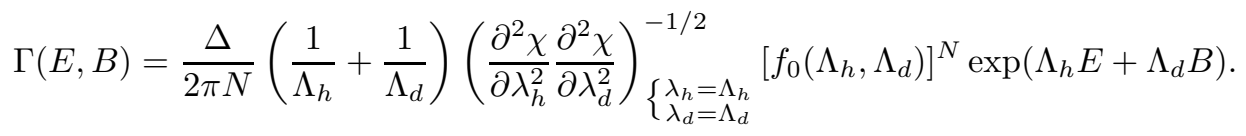

Let us generalize the method proposed by Yu. Krutkov [14] in order to derive the density of distribution for a system in a thermostat. As a model of the thermostat, for which this method is applicable in the case of a gas of $N$ particles in the magnetic field, one can consider a gas of $M$ particles located in a parallel plane at a distance smaller than the radius of interaction, so that particles belonging to different systems are allowed to collide, but there is no particle exchange, and $N \ll M$. The relations $E_{C}=E_{N}+E_{\mathrm{M}}$ and $B_{C}=B_{N}+B_{\mathrm{M}}$ hold, where $E_{C}$ and $B_{C}$ are values of corresponding integrals for the whole system, $C=N+M$. The probability of the subsystem of $N$ particles being located in a chosen phase space element $\delta \Phi_{N}$ situated in a layer bounded by surfaces $\left(E_{N}, B_{N}\right)$ and $\left(E_{N}+\Delta, B_{N}+\Delta\right)$ is given by equation

$$
W=\frac{\Gamma_{\mathrm{M}}\left(E_{C}-E_{N}, B_{C}-B_{N}\right) \delta \Phi_{N}}{\Gamma\left(E_{C}, B_{C}\right)} .
$$

Let us represent $\Gamma_{\mathrm{M}}\left(E_{C}-E_{N}, B_{C}-B_{N}\right)$ as the inverse Laplace transform of (30):

$$
\begin{aligned}
\Gamma_{\mathrm{M}}\left(E_{T}-E_{N}, B_{T}-B_{N}\right)= & \Delta\left(\frac{1}{2 \pi i}\right)^{2} \int_{a_{h}-i \infty}^{a_{h}+i \infty} \mathrm{d} \lambda_{h} \int_{a_{d}-i \infty}^{a_{d}+i \infty} \mathrm{d} \lambda_{d}\left(\frac{1}{\lambda_{h}}+\frac{1}{\lambda_{d}}\right) \\
& \times\left[f_{0}\left(\lambda_{h}, \lambda_{d}\right)\right]^{T-N} \exp \left[\lambda_{h}\left(E_{T}-E_{N}\right)+\lambda_{d}\left(B_{T}-B_{N}\right)\right]
\end{aligned}
$$

and calculate it using the saddle-point method. The saddle-points are determined from the set of equations (31) for the function $\chi_{C}\left(\lambda_{h}, \lambda_{d} ; E_{C}, B_{C}\right)$, and the other quantities are factored out from the integral with values $\lambda_{h}=\Lambda_{h(C)}, \lambda_{d}=\Lambda_{d(C)}$ substituted, since $N \ll C$. Substituting the result into (33) and replacing the values of the integrals of motion with the integrals themselves, we find the density of distribution for an ensemble representing the $N$-subsystem in the form

$$
f_{N}(p, q)=Z^{-1} \exp \left[-\Lambda_{h(C)} \mathrm{H}_{N}(p, q)-\Lambda_{d(C)} \mathrm{D}_{N}(p, q)\right],
$$

where

$Z=\left(N ! h^{2 N}\right)\left[f_{0}\left(\Lambda_{h(C)}, \Lambda_{d(C)}\right)\right]^{N}=\left(N ! h^{2 N}\right) \int_{\Phi_{N}} \exp \left[-\Lambda_{h(C)} \mathrm{H}_{N}(p, q)-\Lambda_{d(C)} \mathrm{D}_{N}(p, q)\right] \mathrm{d} \Phi_{N}$.

From reasoning, outlined, e.g., in [13], a factor $\left(N ! h^{2 N}\right)^{-1}$, where $h$ is the Planck constant, is necessary to be inserted into the normalization factor.

We emphasize that equation (35) was obtained at the condition that the integrals of motion $\mathrm{H}(p, q)$ and $\mathrm{D}(p, q)$ completely define the layer of the phase space containing the system trajectory. It would be incorrect to represent the density of distribution in a form similar to (35) in order to take into account the conservation of any other integral of motion, e.g. $\mathrm{L}_{z}(p, q)$, which does not possess the properties of $\mathrm{D}(p, q)$.

The density of distribution (35) coincides in its form, up to unessential factors, with the density of distribution for the microcanonical ensemble (in the layer where it is other than zero) which can be deduced from (32). An essential difference between them is the determination of the saddle points. For the microcanonical ensemble $\Lambda_{h}$ and $\Lambda_{d}$ are the functions of mean values of the determining integrals $E_{N}$ and $B_{N}$ per one particle, i.e. they are internal parameters of the system, whereas for the system in a thermostat these are external parameters determined by properties of the whole system. Hereafter we shall imply this, omitting subscript $(C)$. This property, once proved for a thermostat with an identical nature to that of the system, is generalized for any kind of thermostat, with $\Lambda_{h}$ identified, as known, with the inverse temperature. 
It is important to clarify the physical meaning of the parameter $\Lambda_{d}$ in the case where the thermostat is not a GMF. For example, if the thermostat is an ordinary ideal gas, there is no parameter measured in units of frequency, determined in its dynamics, and it is impossible to introduce an integral of motion $\mathrm{D}_{\mathrm{M}}$ according to (8) and (21) so that it has a physical meaning. Such an integral can be defined nominally, using the frequency $\omega$ characteristic for the GMF. Then, keeping in mind that the total angular momentum is equal to zero for the thermostat, we derive that $E_{\mathrm{M}}=B_{\mathrm{M}}$ and $\Lambda_{h(\mathrm{M})}=\Lambda_{d(\mathrm{M})}$. Note that though it seems apparent from (34) that the thermodynamics of the GMF is identical to that of the system with the Hamiltonian $H+D$, this is not the case. Though $\mathrm{H}$ and $\mathrm{D}$ are measured in the same units, these integrals have different physical meanings: as it follows from (8) and (21), the $d$-integral characterizes the geometric properties of the system. This is especially obvious for the case of noninteracting particles, when $U \equiv 0$. The heat transfer from one system to another occurs when their particles collide, when the energies and angular momenta of the colliding particles change, but the sums of these values are conserved. If the average energy per particle in the first system is greater than that in the second one, then in the average over a large number of collisions, the first system will transfer the energy to the second one. If the total angular momenta of both systems are zero, the average transfer of the angular momentum is also zero. Thus, the transfer of the average value of the $d$-integral is associated with the process similar to the energy transfer and is equal to the latter numerically, but it determines a change in the density of orbit centers of particles. Throughout the following consideration we shall examine the systems with the average angular momenta equal to zero, so that $\Lambda_{h}=\Lambda_{d}$, and the subscript of $\Lambda$ may be omitted. It can be shown that this quantity possesses the necessary property of transitivity (see, e.g., [17]). Therefore, we can conclude that $\Lambda^{-1}$ is proportional to temperature. The derivation of the density of distribution for a system in a thermostat may be considered more rigorously within the quantum statistical thermodynamics. Then we shell obtain $\Lambda^{-1}=2 T$.

The measurable physical properties are expressed in terms of the derivatives of the free energy $F=-T \ln Z(T, H, N)$ and other thermodynamic potentials. A thermodynamic potential, corresponding to the internal energy of an ordinary gas, is given by the function

$$
(\overline{\mathrm{H}}+\overline{\mathrm{D}})=-T^{2}\left[\frac{\partial}{\partial T}\left(\frac{F}{T}\right)\right]_{H}=\frac{E+B}{2}=E,
$$

i.e. the internal energy of the GMF is considered as a function of entropy and magnetic field. The latter equality in (36) is caused by the fact that the exact value of the angular momentum of the total system (GMF plus thermostat) is assumed to be zero, while the average value of the angular momentum of the GMF is also zero. From equation (36) and further on, $E$ and $B$ denote the ensemble averages of the corresponding integrals. The entropy of the GMF is written as

$$
S=-\int_{\Phi_{N}} f(p, q) \ln f(p, q) \mathrm{d} \Phi=\frac{1}{T}\left(\frac{E+B}{2}-F\right)=\frac{1}{T}(E-F) .
$$

The set of the physical properties itself is changed. The state of the GMF, exposed to an external potential field, depends on a set of parameters determining the state. In the case of a reversible change of these parameters, a change of state can be described by introducing corresponding generalized forces. In particular, the area in which the GMF is bounded by the potential barrier can be considered as one of such parameters, while the pressure applied to the barrier is the corresponding force. But the existence of both an external potential field and such parameters is not mandatory for the equilibrium state of the GMF to be established. Instead, the magnetic field is the mandatory parameter, and the generalized magnetic moment given by

$$
-\left[\frac{\partial(E+B)}{\partial H}\right]_{S}=\frac{M_{z(h)}+M_{z(d)}}{2}=M_{z}=-\left[\frac{\partial E}{\partial H}\right]_{S},
$$

is the corresponding force. Here $M_{z}$ is the ordinary magnetic moment of the GMF.

From equation (19) and from the density of distribution (35) expressed in the coordinates (10) one can see that, in the absence of the potential field, we would get $M_{z}=-E / H$ in complete 
agreement with expression (13) for the average magnetic moment of one particle. Therefore, we overcome the paradoxical result of the Bohr - van Leeuwen theorem [1-3] asserting that, though the magnetic moment of each particle, averaged over the period, is given by equation (13) the total magnetic moment of the GMF is equal to zero (see section 1). The repulsion of particles being taken into account by the mean field method gives rise to a force acting on each particle and directed outward from the center. The drift (6) is responsible for the creation of additional diamagnetism. On the other hand, the presence of a neutralizing charge, distributed over a finite area, gives rise to a centrally-directed force acting on the particles located near the boundary of this area. The drift of these particles creates a current generating a paramagnetic moment. The result of summation of both moments depends on the number of particles in the GMF, temperature, and the parameters determining the density of the neutralizing charge.

It is noteworthy that the formulae deduced for the GMF in the absence of the bounding barrier do not allow one to obtain corresponding expressions for a gas in the absence of a magnetic field by putting $H \rightarrow 0$. This operation cancels the restriction of the phase volume in the coordinate subspace, and the gas becomes unstable.

It follows from (15), (17) and (37) that

$$
\mathrm{d} E=T \mathrm{~d} S-M_{z} \mathrm{~d} H .
$$

This equality is formally similar to the thermodynamic relation $\mathrm{d} E=T \mathrm{~d} S-P \mathrm{~d} V$, but the equality of the magnetic moments of the systems in the equilibrium does not follow from (39) because the magnetic field is the same in both systems and changes by the same value.

Now we move from further construction of the thermodynamics of the GMF, to the consideration of the issues of the density of particles and the density of the electric current generating the magnetic moment. The density of particles $\sigma(\mathbf{r})$ at the point $\mathbf{r}$ of the plane $z=0$ can be evaluated as an average from the expression

$$
\sigma(\mathbf{r})=\sum_{\nu} \delta\left(\mathbf{r}-\mathbf{r}_{\nu}\right)
$$

over the distribution (35) with $\Lambda_{h(C)}=\Lambda_{d(C)}=(2 T)^{-1}$. Here $\delta(\mathbf{r})$ is Dirac's delta function.

If the Coulomb field is described by a self-consistent field, we get

$$
\sigma(\mathbf{r})=\frac{N}{2 \pi}\left\{\int_{0}^{\infty} \exp \left[-\frac{1}{T}\left(\frac{m \omega^{2} r^{2}}{8}+u(r)\right)\right] r \mathrm{~d} r\right\}^{-1} \exp \left[-\frac{1}{T}\left(\frac{m \omega^{2} r^{2}}{8}+u(r)\right)\right],
$$

while the potential energy of a particle in the self-consistent field is determined by a nonlinear integral equation given by

$$
u(r)=-\mathrm{e} \int_{\Omega} \frac{\rho_{+}\left(\mathbf{r}^{\prime}\right)-\mathrm{e} \sigma\left(\mathbf{r}^{\prime}\right)}{\left|\mathbf{r}-\mathbf{r}^{\prime}\right|} \mathrm{d} \Omega+u_{0}
$$

Here the integration is made over the whole plane $\Omega, u_{0}>0$ is a constant providing non-negativity of $u(r), \rho_{+}(\mathbf{r})$ is the density of the positive neutralizing charge, which is also assumed to be axially symmetric. If this density is homogeneous within a circle of radius $R_{N}$ and is zero outside it, then the potential energy increases abruptly at $r \simeq R_{N}$. At sufficiently large $r$, the first term makes the chief contribution to the exponent in equation (41), resulting in the exponential decay of the density of the GMF at large distances from its mass center. The potential energy (42) is a function of the number of particles $N$ and the radius $R_{N}$ of the area filled with the positive charge. Therefore, the density of particles is not just proportional to $N$, but also depends on these parameters in a complicated way. The first term in the exponent in equation (41) is proportional to $H^{2}$ so that the width of the distribution of particles on the plane decreases as the magnetic field increases. It also decreases as the temperature decreases.

If we average (40) with the ordinary density of distribution (1) and change the integration variables according to (4), we get the equation (41) for the surface density of particles, but with the first term missing in the exponent. Let us restrict the region of integration $\Omega$ by a circle of radius $R_{N}$. Then it represents the system volume, i.e. an area which the particles cannot leave. If 
the density $\rho_{+}(\mathbf{r})$ is homogeneous within this circle, then a self-consistent solution of equation (42) is given by $u(r) \equiv 0, \sigma(r) \equiv \rho_{+} /$e everywhere at $r<R_{N}$. This fact justifies the frequently used model of non-interacting particles in a potential box. However, as shown in this work, the density of distribution (1) is not suitable to describe the properties of the GMF. If the density of distribution (35) is employed, the model of non-interacting particles in a potential box can be considered as the zero approximation with respect to the parameter $m \omega^{2} R_{N}^{2} / 8 T$. For free electrons, this means that $R_{N}^{2} H^{2} / 8 T_{\mathrm{K}} \ll 3.2 \cdot 10^{-4}\left(\mathrm{~A}^{2} / \mathrm{K}\right)$, where $T_{\mathrm{K}}$ is the temperature measured in Kelvin degrees. This inequality can be realized. For a large radius, low temperature, and strong magnetic field, the effect reducing the density of a nondegenerate electron gas should be observed in a film area near the boundary.

The current density at a point $\mathbf{r}$ is given by the ensemble average of the expression

$$
\mathbf{j}=-\frac{e}{\delta z} \sum_{\nu} \delta\left(\mathbf{r}-\mathbf{r}_{\nu}\right) \mathbf{v}_{\nu}
$$

Here $\delta z$ is a "thickness" of particles introduced in order to use the formulae of three-dimensional electro-dynamics. Van Vleck [4] noted that, if all positions of the orbits with the same energy (radius) are equiprobable, then there are two orbits passing through each point, centrally symmetrical about it and generating oppositely directed currents. In the case of an ensemble described by the density of distribution (35), the equal probabilities of the orbits are not realized, and the current density cannot be identically equal to zero. From the symmetry reasoning, there exists only the azimuthally directed current density depending on the distance from the center. Then the vector differential equation, linking the current density and the magnetization $\mu_{z}(r)$ reduces to a single first order equation

$$
-\frac{\mathrm{d} \mu_{z}}{\mathrm{~d} r}=\frac{1}{c} j_{\varphi}(r)
$$

with the solution

$$
\mu_{z}(r)=\frac{1}{c} \int_{r}^{\infty} j_{\varphi}(\rho) \mathrm{d} \rho .
$$

The magnetization field defined by (45) is not identically equal to zero and is not uniform. The magnetic moment of the GMF is given by

$$
M_{z}(r)=\frac{\pi \delta z}{c} \int_{0}^{\infty} \rho^{2} j_{\varphi}(\rho) \mathrm{d} \rho,
$$

with the "thickness" cancelled.

\section{Conclusion}

The results of this study suggest that the description of properties of the GMF should be based upon the density of distribution properly taking into account the conservation of a $d$-integral, or, in the case of the quantum-mechanical system, a corresponding statistical operator. As a first example, we demonstrated that the physically odd result of the Bohr-van Leeuwen theorem is wrong, because this theorem proceeds from an incorrect assumption. During the past seventy years, numerous studies concerning the properties of the GMF have been carried out based on the equations deduced in [12] (see, for example, $[7,9]$ ). Other studies were based directly on the quantum analogue of the density of distribution (1) and the Fermi distribution (see, for example, [19] and further investigations on this subject). We claim that all these works require a revision.

\section{References}

1. Bohr N., Nature (London), 1991, 88, 200.

2. Bohr N. Dissertation. Copenhagen, 1911 (unpublished).

3. van Leeuwen J.H., J. de Phys., 1921, 2, No. 6, 361. 
4. van Vleck J.H. Theory of Electric and Magnetic Susceptibilities. Oxford University Press, 1932, reprinted in 1965.

5. Peierls R.E. Quantum Theory of Solids. Clarendon Press, Oxford, 1955.

6. Landau L.D., Lifshitz E.M. Statistical Physics, 3rd rev., part I. Pergamon Press, New York, 1980.

7. Lifshits I.M., Azbel M.J., Kaganov M.I. Electron Theory for Metals. Nauka, Moscow, 1971.

8. White R.M. Quantum Theory of magnetism. Springer Verlag, Berlin, 1983.

9. Shoenberg D. Magnetic Oscillations in Metals. Cambridge University Press, Cambridge, 1984.

10. Abrikosov A.A. Principles of the Theory of Metals. Nauka, Moscow, 1987.

11. Magnetic Properties of Matter, edited by F. Borsa and V. Tognetti. World Scientific, Singapore, New Jersey, Hong Kong, 1988.

12. Landau L.D., Zs. Phys., 1930, 64, 629.

13. Zubarev D.N. Nonequilibrium Statistical Thermodynamics. Nauka, Moscow, 1971.

14. Krutkov Yu.A., Zs. Phys., 1933, 81, 377.

15. Debye P., Mitteilungen d. Phys. Ges. Zurich, 1916, No. 18.

16. Kubo R. Statistical Mechanics. North-Holland Publishing Company, Amsterdam, 1965.

17. Hinchin A.Ya. Mathematical Principles of Statistical Mechanics. GITTL, Moscow-Leningrad, 1943.

18. Kubo R. Thermodynamics. North-Holland Publishing Company, Amsterdam, 1968.

19. The Quantum Hall Effect, edited by Richard E. Prange and Steven M. Girvin. Springer Verlag, Berlin, Heidelberg, London, Paris, Tokyo, 1987.

\title{
Класична статистична термодинаміка газу заряджених частинок у магнітному полі
}

\author{
І.М.Дубровський \\ Інститут металофізики, \\ бульв. Вернадського 36, \\ Київ-142, 03680, Україна \\ Отримано 4 липня 2005 р.
}

Показано, що парадокс класичної статистичної термодинаміки для газу заряджених частинок у магнітному полі (ГМП) не було роз'яснено. Парадокс полягає у твердженні, що середній магнітний момент газу дорівнює нулю, тоді як середне по часу від магнітного моменту кожної частинки завжди від'ємне. Розглянуто газ заряджених частинок, що рухається у площині перпендикулярній магнітному полю. Густина розподілу ансамблю, що описує статистичні властивості ГМП виведена, починаючи з основ, беручи до уваги специфічний характер динаміки заряджених частинок у магнітному полі. Підкреслено, що ні введення потенціального бар'єру, що обмежує область існування ГМП, ні введення фону нейтралізуючого заряду, що займає обмежену площину, не є необхідними умовами для існування стаціонарного рівноважного стану ГМП. Показано, що причиною цього факту $\epsilon$ залежність густини розподілу ансамблю від ще одного, крім гамільтоніану, додатньо визначеного інтегралу руху, що є лінійною комбінацією гамільтоніану і кутового моменту ГМП. Головні термодинамічні рівняння виведені з нової густини розподілу, і показано, що ГМП має магнітний момент, величина і знак якого визначаються зовнішнім потенціальним полем. Зокрема, ГМП є діамагнітним, якщо нейтралізуючий фон відсутній. Таким чином, твердження теореми Бора-ван Леєвен, виведене із застосуванням звичайної густини розподілу Гіббса, що залежить тільки від гамільтоніану, є хибним. Відзначається, що багато робіт з теорії електронних явищ у магнітному полі базуються на тій самій хибній густині розподілу або на формулі середніх чисел заповнення, залежних від енергії станів, що витікає з цієї густини розподілу у квантовій теорії. Ці теорії повинні бути переглянуті у світлі нової густини розподілу.

Ключові слова: газ, заряджені частинки, магнітне поле, густина розподілу, фазовий простір, інтеграл руху, термодинаміка, магнітний момент

PACS: $05.20 . \mathrm{Gg}, 75.20 .-\mathrm{g}$ 